\title{
Erratum to "Slender Sheath/Guiding Catheter Combination vs. Sheathless Guiding Catheter for Acute Coronary Syndrome: A Propensity-Matched Analysis of the Two Devices"
}

\author{
Tsuyoshi Isawa $\mathbb{D}^{\mathbb{1}},{ }^{1}$ Kazunori Horie, ${ }^{1}$ Taku Honda, ${ }^{1}$ Masataka Taguri, ${ }^{2}$ and Norio Tada $^{1}$ \\ ${ }^{1}$ Department of Cardiology, Sendai Kousei Hospital, Sendai, Japan \\ ${ }^{2}$ Department of Data Science, Yokohama City University School of Data Science, Yokohama, Japan
}

Correspondence should be addressed to Tsuyoshi Isawa; isa_tsuyo@yahoo.co.jp

Received 29 September 2020; Accepted 29 September 2020; Published 23 October 2020

Copyright (c) 2020 Tsuyoshi Isawa et al. This is an open access article distributed under the Creative Commons Attribution License, which permits unrestricted use, distribution, and reproduction in any medium, provided the original work is properly cited.

In the article titled "Slender Sheath/Guiding Catheter Combination vs. Sheathless Guiding Catheterfor Acute Coronary Syndrome: A Propensity-Matched Analysis of the Two Devices" [1], there were errors in Tables 2, 3, and 4. In each of these tables the $n$ value was incorrectly given as $n=397$ for the propensity-matched population in both the Glidesheath and Sheathless columns. The correct value is $n=364$. This mistake occurred during the production of the article, and the publisher apologises for this error.

The corrected tables are shown below and are listed as Tables $2-4$, respectively.

TABle 2: Procedural characteristics of the study population.

\begin{tabular}{|c|c|c|c|c|c|c|}
\hline \multirow[b]{2}{*}{ Variables } & \multicolumn{3}{|c|}{ Total population } & \multicolumn{3}{|c|}{ Propensity-matched population } \\
\hline & $\begin{array}{c}\text { Glidesheath } \\
n=397\end{array}$ & $\begin{array}{c}\text { Sheathless } \\
n=711\end{array}$ & $P$ & $\begin{array}{c}\text { Glidesheath } \\
n=364\end{array}$ & $\begin{array}{c}\text { Sheathless } \\
n=364\end{array}$ & $P$ \\
\hline LAD/diagonal, $n(\%)$ & $196(49.4)$ & $318(44.7)$ & 0.15 & $179(49.2)$ & $170(46.7)$ & 0.55 \\
\hline LCX/marginal, $n(\%)$ & $37(9.3)$ & $144(20.3)$ & $<0.001$ & $33(9.1)$ & $72(19.8)$ & $<0.001$ \\
\hline $\mathrm{RCA}, n(\%)$ & $139(35.0)$ & $217(30.5)$ & 0.14 & $132(36.3)$ & $109(30.0)$ & 0.08 \\
\hline LMCA, $n(\%)$ & $25(6.3)$ & $32(4.5)$ & 0.20 & $20(5.5)$ & $13(3.6)$ & 0.29 \\
\hline \multicolumn{7}{|l|}{ Guiding catheter type } \\
\hline LAD/diagonal (JL/EBU/others), $n$ (\%) & $\begin{array}{c}143 \\
(73.0) / 51 \\
(26.0) / 2(1.0)\end{array}$ & $\begin{array}{c}263 \\
(82.7) / 54 \\
(17.0) / 1 \\
(0.3)\end{array}$ & 0.025 & $\begin{array}{c}132 \\
(73.7) / 46 \\
(25.7) / 1 \\
(0.6)\end{array}$ & $\begin{array}{c}144 \\
(84.7) / 26 \\
(15.3) / 0(0)\end{array}$ & 0.033 \\
\hline $\mathrm{LCX} /$ marginal (JL/EBU/others), $n(\%)$ & $\begin{array}{c}13 \\
(35.1) / 23 \\
(62.2) / 1 \\
(2.7)\end{array}$ & $\begin{array}{c}79 \\
(54.9) / 65 \\
(45.1) / 0 \\
(0)\end{array}$ & 0.019 & $\begin{array}{c}12 \\
(36.4) / 20 \\
(60.6) / 1 \\
(3.0)\end{array}$ & $\begin{array}{c}42 \\
(58.3) / 30 \\
(41.7) / 0(0)\end{array}$ & 0.050 \\
\hline RCA (JR/AL/others), $n(\%)$ & $\begin{array}{c}101 \\
(72.7) / 30 \\
(21.6) / 8 \\
(5.7)\end{array}$ & $\begin{array}{c}157 \\
(72.4) / 58 \\
(26.7) / 2 \\
(0.9)\end{array}$ & 0.018 & $\begin{array}{c}97 \\
(73.5) / 28 \\
(21.2) / 7 \\
(5.3)\end{array}$ & $\begin{array}{c}81 \\
(74.3) / 26 \\
(23.9) / 2(1.8)\end{array}$ & 0.22 \\
\hline
\end{tabular}


TABLE 2: Continued.

\begin{tabular}{|c|c|c|c|c|c|c|}
\hline \multirow[b]{2}{*}{ Variables } & \multicolumn{3}{|c|}{ Total population } & \multicolumn{3}{|c|}{ Propensity-matched population } \\
\hline & $\begin{array}{c}\text { Glidesheath } \\
n=397\end{array}$ & $\begin{array}{c}\text { Sheathless } \\
n=711\end{array}$ & $P$ & $\begin{array}{c}\text { Glidesheath } \\
n=364\end{array}$ & $\begin{array}{c}\text { Sheathless } \\
n=364\end{array}$ & $P$ \\
\hline LMCA (JL/EBU/others), $n$ (\%) & $\begin{array}{c}15 \\
(60.0) / 9 \\
(36.0) / 1 \\
(4.0)\end{array}$ & $\begin{array}{c}28 \\
(87.5) / 3 \\
(9.4) / 1 \\
(3.1)\end{array}$ & 0.0046 & $\begin{array}{c}14 \\
(70.0) / 6 \\
(30.0) / 0 \\
(0)\end{array}$ & $\begin{array}{c}12 \\
(92.3) / 1 \\
(7.7) / 0 \\
(0)\end{array}$ & 0.12 \\
\hline True bifurcation lesion, $n(\%)$ & $67(16.9)$ & $99(13.9)$ & 0.19 & $58(15.9)$ & $51(14.0)$ & 0.53 \\
\hline In-stent restenosis/occlusion, $n(\%)$ & $26(6.6)$ & $39(5.5)$ & 0.58 & $24(6.6)$ & $18(5.0)$ & 0.43 \\
\hline Diffuse lesion, $n(\%)$ & $264(66.5)$ & $434(61.0)$ & 0.080 & $246(67.6)$ & $228(62.6)$ & 0.19 \\
\hline Heavily calcified lesion, $n$ (\%) & $13(3.3)$ & $10(1.4)$ & 0.047 & $12(3.3)$ & $5(1.4)$ & 0.14 \\
\hline Thrombus aspiration, $n(\%)$ & $218(54.9)$ & $253(35.6)$ & $<0.001$ & $203(55.8)$ & $135(37.1)$ & $<0.001$ \\
\hline Rotablation, $n(\%)$ & $8(2.0)$ & $6(0.8)$ & 0.16 & $7(1.9)$ & $2(0.6)$ & 0.18 \\
\hline Arterial access site & & & & & & \\
\hline Left radial, $n(\%)$ & $373(94.0)$ & $679(95.5)$ & 0.26 & $344(94.5)$ & $343(94.2)$ & 1.00 \\
\hline $\begin{array}{l}\text { No. of previous iTRA attempts, median } \\
(\min -\max )\end{array}$ & $0(0-9)$ & $0(0-11)$ & 0.002 & $0(0-9)$ & $0(0-11)$ & 0.17 \\
\hline $\begin{array}{l}\text { No. of previous iTRI attempts, median } \\
\text { (min-max) }\end{array}$ & $0(0-7)$ & $0(0-7)$ & 0.059 & $0(0-7)$ & $0(0-7)$ & 0.36 \\
\hline
\end{tabular}

Data are presented as median (interquartile range) or $n$ (\%), unless otherwise indicated. "Glidesheath" denotes 7-Fr Glidesheath slender/7-Fr guiding catheter combination group, and "Sheathless" denotes 7.5-Fr sheathless guiding catheter group. The asterisk denotes a statistically significant difference between the two groups. AL, Amplatz type; EBU, extra backup type; iTRA, ipsilateral transradial coronary angiography; iTRI, ipsilateral transradial coronary intervention; JL, Judkins Left type; JR, Judkins Right type; LAD, left anterior descending coronary artery; LCX, left circumflex coronary artery; LMCA, left main coronary artery; RCA, right coronary artery.

TABLE 3: Procedural outcomes.

\begin{tabular}{|c|c|c|c|c|c|c|c|c|}
\hline \multirow[b]{2}{*}{ Variables } & \multicolumn{4}{|c|}{ Total population } & \multicolumn{4}{|c|}{ Propensity-matched population } \\
\hline & $\begin{array}{c}\text { Glidesheath } \\
n=397\end{array}$ & $\begin{array}{c}\text { Sheathless } \\
n=711\end{array}$ & OR $(95 \% \mathrm{CI})$ & $P$ & $\begin{array}{l}\text { Glidesheath } \\
n=364\end{array}$ & $\begin{array}{c}\text { Sheathless } \\
n=364\end{array}$ & OR $(95 \% \mathrm{CI})$ & $P$ \\
\hline $\begin{array}{l}\text { Procedural success, } n \\
(\%)\end{array}$ & $392(98.7)$ & $702(98.7)$ & $1.00(0.99-1.01)$ & 1.0 & $359(98.6)$ & $361(99.2)$ & $0.99(0.98-1.01)$ & 0.73 \\
\hline $\begin{array}{l}\text { Coronary ostial } \\
\text { dissection, } n(\%)\end{array}$ & $3(0.8)$ & $10(1.4)$ & $1.86(0.52-6.72)$ & 0.40 & $3(0.8)$ & $6(1.7)$ & $0.50(0.13-1.98)$ & 0.51 \\
\hline $\begin{array}{l}\text { Access-site crossover } \\
\text { from radial to femoral, } \\
n(\%)\end{array}$ & $1(0.3)$ & $0(0)$ & $\mathrm{n} / \mathrm{a}$ & 0.36 & $1(0.3)$ & $0(0)$ & $\mathrm{n} / \mathrm{a}$ & 1.00 \\
\hline $\begin{array}{l}\text { Total fluoroscopy } \\
\text { time, min }\end{array}$ & $22.4(15.3-31.5)$ & $\begin{array}{c}18.2 \\
(13.9-28.0)\end{array}$ & & $<0.001$ & $\begin{array}{c}22.3 \\
(15.3-31.5)\end{array}$ & $\begin{array}{c}18.7 \\
(13.8-29.5)\end{array}$ & & 0.002 \\
\hline Contrast used, ml & $128(100-160)$ & $\begin{array}{c}127 \\
(103-160)\end{array}$ & & 0.67 & $128(100-160)$ & $\begin{array}{c}133 \\
(105-165)\end{array}$ & & 0.11 \\
\hline $\begin{array}{l}\text { No. of catheters used, } \\
\text { median (min-max) }\end{array}$ & $1(1-5)$ & $1(1-4)$ & & $<0.001$ & $1(1-5)$ & $1(1-4)$ & & 0.016 \\
\hline
\end{tabular}

Data are presented as median (interquartile range) or $n$ (\%), unless otherwise indicated. "Glidesheath" denotes 7-Fr Glidesheath slender/7-Fr guiding catheter combination group, and "Sheathless" denotes 7.5-Fr sheathless guiding catheter group. The asterisk denotes a statistically significant difference between the two groups. CI, confidence interval; n/a, not applicable; OR, odds ratio.

TABle 4: Periprocedural access-site complications.

\begin{tabular}{|c|c|c|c|c|c|c|c|c|}
\hline \multirow[b]{2}{*}{ Variables } & \multicolumn{4}{|c|}{ Total population } & \multicolumn{4}{|c|}{ Propensity-matched population } \\
\hline & $\begin{array}{c}\text { Glidesheath } \\
n=397\end{array}$ & $\begin{array}{c}\text { Sheathless } \\
n=711\end{array}$ & OR $(95 \% \mathrm{CI})$ & $P$ & $\begin{array}{c}\text { Glidesheath } \\
n=364\end{array}$ & $\begin{array}{c}\text { Sheathless } \\
n=364\end{array}$ & OR $(95 \% \mathrm{CI})$ & $P$ \\
\hline $\begin{array}{l}\text { RAD at } 30 \text { days, } \\
\mathrm{mm}\end{array}$ & $2.0(1.8-2.3)$ & $2.1(1.8-2.4)$ & & 0.15 & $2.0(1.8-2.3)$ & $2.1(1.8-2.4)$ & & 0.06 \\
\hline $\begin{array}{l}\text { End-procedural } \\
\text { ACT, s }\end{array}$ & $273(231-312)$ & $301(250-376)$ & & $<0.001$ & $273(229-310)$ & $289(250-372)$ & & $<0.001$ \\
\hline $\begin{array}{l}\text { RAO at } 30 \text { days, } n \\
(\%)\end{array}$ & $6(1.5)$ & $25(3.5)$ & $0.43(0.18-1.04)$ & 0.058 & $5(1.4)$ & $15(4.1)$ & $0.33(0.12-0.91)$ & 0.039 \\
\hline
\end{tabular}


TABLE 4: Continued.

\begin{tabular}{|c|c|c|c|c|c|c|c|c|}
\hline \multirow[b]{2}{*}{ Variables } & \multicolumn{4}{|c|}{ Total population } & \multicolumn{4}{|c|}{ Propensity-matched population } \\
\hline & $\begin{array}{c}\text { Glidesheath } \\
n=397\end{array}$ & $\begin{array}{c}\text { Sheathless } \\
n=711\end{array}$ & OR $(95 \% \mathrm{CI})$ & $P$ & $\begin{array}{c}\text { Glidesheath } \\
n=364\end{array}$ & $\begin{array}{c}\text { Sheathless } \\
n=364\end{array}$ & OR $(95 \% \mathrm{CI})$ & $P$ \\
\hline $\begin{array}{l}\text { Severe radial } \\
\text { spasm, } n(\%) \\
\end{array}$ & $9(2.3)$ & $11(1.6)$ & $1.46(0.61-3.49)$ & 0.48 & $5(1.4)$ & $7(1.9)$ & $0.71(0.23-2.22)$ & 0.58 \\
\hline $\begin{array}{l}\text { Access-site major } \\
\text { bleeding within } 30 \\
\text { days } \\
\text { BARC type } 3 \text { or } 5 \text {, } \\
n(\%)\end{array}$ & $5(1.3)$ & $9(1.3)$ & $0.99(0.34-2.95)$ & 1.00 & $5(1.4)$ & $6(1.6)$ & $0.83(0.26-2.71)$ & 1.00 \\
\hline $\begin{array}{l}\text { BARC type } 3, n \\
(\%)\end{array}$ & $5(1.3)$ & $9(1.3)$ & $0.99(0.34-2.95)$ & 1.00 & $5(1.4)$ & $6(1.6)$ & $0.83(0.26-2.71)$ & 1.00 \\
\hline $\begin{array}{l}\text { BARC type } 5, n \\
(\%)\end{array}$ & $0(0)$ & $0(0)$ & $\mathrm{n} / \mathrm{a}$ & $\mathrm{n} / \mathrm{a}$ & $0(0)$ & $0(0)$ & $\mathrm{n} / \mathrm{a}$ & $\mathrm{n} / \mathrm{a}$ \\
\hline
\end{tabular}

Data are presented as median (interquartile range) or $n$ (\%), unless otherwise indicated. "Glidesheath" denotes 7-Fr Glidesheath slender/7-Fr guiding catheter combination group, and "Sheathless" denotes 7.5-Fr sheathless guiding catheter group. The asterisk denotes a statistically significant difference between the two groups. ACT; activated clotting time; BARC, bleeding academic research consortium; CI, confidence interval; n/a, not applicable; OR, odds ratio; RAD, radial artery diameter; RAO, radial artery occlusion.

\section{References}

[1] T. Isawa, K. Horie, T. Honda, M. Taguri, and N. Tada, "Slender sheath/guiding catheter combination vs. sheathless guiding catheter for acute coronary syndrome: a propensity-matched analysis of the two devices," Journal of Interventional Cardiology, vol. 2020, Article ID 8216831, 10 pages, 2020. 